\title{
Lipid nanostructures for antioxidant delivery: a comparative preformulation study
}

\author{
Elisabetta Esposito ${ }^{* 1}$, Maddalena Sguizzato ${ }^{1}$, Markus Drechsler ${ }^{2}$, Paolo Mariani ${ }^{3}$, \\ Federica Carducci ${ }^{3}$, Claudio Nastruzzi ${ }^{1}$, Giuseppe Valacchi ${ }^{4,5,6}$ and Rita Cortesi ${ }^{* 1}$
}

\section{Full Research Paper}

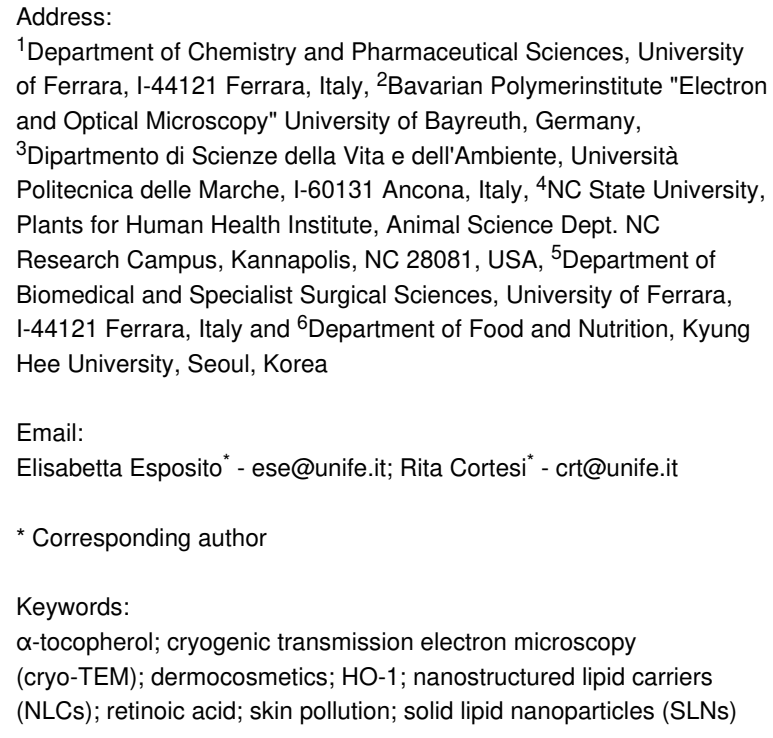

${ }^{1}$ Department of Chemistry and Pharmaceutical Sciences, University of Ferrara, I-44121 Ferrara, Italy, ${ }^{2}$ Bavarian Polymerinstitute "Electron and Optical Microscopy" University of Bayreuth, Germany, ${ }^{3}$ Dipartmento di Scienze della Vita e dell'Ambiente, Università Politecnica delle Marche, I-60131 Ancona, Italy, ${ }^{4}$ NC State University, Plants for Human Health Institute, Animal Science Dept. NC Research Campus, Kannapolis, NC 28081, USA, ${ }^{5}$ Department of Biomedical and Specialist Surgical Sciences, University of Ferrara, I-44121 Ferrara, Italy and ${ }^{6}$ Department of Food and Nutrition, Kyung Hee University, Seoul, Korea

Email:

Elisabetta Esposito* - ese@unife.it; Rita Cortesi - crt@unife.it

* Corresponding author

\section{Keywords:}

$\alpha$-tocopherol; cryogenic transmission electron microscopy (cryo-TEM); dermocosmetics; HO-1; nanostructured lipid carriers (NLCs); retinoic acid; skin pollution; solid lipid nanoparticles (SLNs)

Beilstein J. Nanotechnol. 2019, 10, 1789-1801. doi:10.3762/bjnano.10.174

Received: 31 May 2019

Accepted: 07 August 2019

Published: 29 August 2019

This article is part of the thematic issue "Frontiers in pharmaceutical nanotechnology".

Guest Editor: M. G. Wacker

(C) 2019 Esposito et al.; licensee Beilstein-Institut. License and terms: see end of document.

\begin{abstract}
This investigation is a study of new lipid nanoparticles for cutaneous antioxidant delivery. Several molecules, such as $\alpha$-tocopherol and retinoic acid, have been shown to improve skin condition and even counteract the effects of exogenous stress factors such as smoking on skin aging. This work describes the design and development of lipid nanoparticles containing antioxidant agents $(\alpha$-tocopherol or retinoic acid) to protect human skin against pollutants. Namely, solid lipid nanoparticles and nanostructured lipid carriers were prepared using different lipids (tristearin, compritol, precirol or suppocire) in the presence or absence of caprylic/ capric triglycerides. The formulations were characterized by particle size analysis, cryogenic transmission electron microscopy, small-angle X-ray diffraction, encapsulation efficiency, preliminary stability, in vitro cytotoxicity and protection against cigarette smoke. Nanostructured lipid carriers were found to reduce agglomerate formation and provided better dimensional stability, as compared to solid lipid nanoparticles, suggesting their suitability for antioxidant loading. Based on the preformulation study, tristearin-based nanostructured lipid carriers loaded with $\alpha$-tocopherol were selected for ex vivo studies since they displayed superior physico-chemical properties as compared to the other nanostructured lipid carriers compositions. Human skin explants were treated with $\alpha$-tocopherol-loaded nanostructured lipid carriers and then exposed to cigarette smoke, and the protein levels of the stress-induced enzyme heme oxygenase were analyzed in skin homogenates. Interestingly, it was found that pretreatment with the nanofor-
\end{abstract}


mulation resulted in significantly reduced heme oxygenase upregulation as compared to control samples, suggesting a protective effect provided by the nanoparticles.

\section{Introduction}

Air pollution increasingly affects industrialized urban areas in a negative manner with dramatic consequences for the environment and human health. This problem also affects rural areas, worsening the air quality all over the world. Besides being the primary cause of many respiratory diseases (e.g., chronic obstructive pulmonary disease, asthma and lung cancer), pollution is also responsible for cutaneous pathologies, spanning from skin aging, inflammation and allergy to skin cancer [1].

Cigarette smoke (CS) is one of the major toxic pollutants, exerting an important role in the onset of many serious and fatal diseases. Indeed, it is well known that CS can provoke various pathologies especially related to the lungs (e.g., cancer, emphysema, bronchitis) as well as the cardiovascular apparatus [2,3] In the last two decades, the noxious effect of CS on skin has been well demonstrated [4-6]. For instance, the chronic exposure of skin to CS induces premature skin ageing, delayed wound healing, psoriasis and inflammatory skin diseases [3]. CS increases the risk of squamous cell carcinoma, with respect to non-smokers, as well as oral leukoplakia and oral cancers, such as lip cancer. Indeed, tobacco smoke is constituted of thousands of toxic compounds, including benzene, formaldehyde, hydrogen cyanide, carbon monoxide, arsenic and radioactive components, producing free radicals that cause oxidative stress [7]. The release of reactive oxygen species from tobacco smoke provokes a series of systemic immunomodulatory effects that leads to a compromised inflammatory response. These destructive mechanisms also affect collagen synthesis and the skin cellular reparative effects $[8,9]$. It has been found that antioxidants play a key role in the regulation of the deleterious activity exerted by CS in humans, nevertheless CS alters the requirements of antioxidants, such as vitamins E and A [9-14]. In this respect, quitting smoking does not always resolve the issue, since even more toxic effects have been shown from exposure to second-hand smoke.

Recently many cosmetic producers have focused their efforts towards antipollution dermocosmetics that are able to defend the skin against prolonged and repetitive daily exposure to pollutants; for instance, film-formers or skin rejuvenating excipients have been developed. Nevertheless, these strategies offer merely a short-term improvement of skin barrier function. Thus, in this respect, there is an unmet need for an effective product that endows skin protection from pollutants from longterm exposure, as well as for antipollution test methods suitable for assessing product efficacy and safety [15].
Vitamin $\mathrm{E}$ is a potent antioxidant, able to counteract the reactive oxygen species production during fat oxidation and free radical propagation - indeed it can protect the cell membranes from free radical attack, acting against lipid peroxidation. Vitamin E exists in 8 different forms, 4 tocopherols and 4 tocotrienols [16]. Among them $\alpha$-tocopherol (TOC) can be mostly adsorbed and accumulated, thus it is largely employed as an antioxidant for edible oils and in anti-aging products. Notably, TOC has been proposed for the treatment of cancer and skin barrier improvement [17-19].

Vitamin A is defined as a group of lipophilic retinoids, including retinoic acid (RA), derived from food and stored in the liver. Due to its antioxidant action, RA plays a role in cancer chemoprevention and differentiation [20]. Particularly, RA has been proposed in the treatment of breast, lung and liver cancers $[21,22]$. Notably, it has been demonstrated that CS induces RA deficiency [23].

Despite the enormous potential of TOC and RA, some drawbacks are associated to their topical use, such as photodegradation, poor water solubility and irritative skin effects when employed in high dosage [24,25]. Thus, TOC and RA need to be loaded in specialized formulations suitable for skin application and able to adequately protect them from degradation. In this respect, recently different lipid nanoparticles have been proposed, including solid lipid nanoparticles (SLNs) and nanostructured lipid carriers (NLCs) [26-28]. SLNs possess several advantages over conventional lipid formulations being able to carry drugs in a biocompatible solid nanometric matrix, thus achieving

1. improvement of solubility,

2. stability of the loaded active molecule and

3. suitability of administration through different routes [29,30].

NLCs represent a smart generation of lipid nanoparticles, being based on a blend of solid and liquid lipids that creates a disordered nano-matrix, able to load higher amounts of lipophilic molecules than SLNs and avoiding leakage during storage [3134].

The choice of the type and concentration of the nanoparticle lipid matrix is crucial since it can affect the physico-chemical aspects of SLNs and NLCs, the encapsulation parameters, as 
well as the long-term stability of the formulation. Thus, in view of an industrial production, a preliminary formulation screening appears imperative [35,36].

The present investigation has been conducted to develop a nanoparticulate approach for counteracting skin pollution. In particular, a preformulation study was performed to select the type and composition of lipid nanoparticles suitable for encapsulation of TOC and RA. To assess the effect of antioxidant loaded in nanoparticles, a Western blot analysis has been performed to evaluate heme oxygenase expression on human skin explants treated with nanoparticles and exposed to CS.

\section{Experimental}

\section{Reagents}

The copolymer poly(ethylene oxide) (80)-poly(propylene oxide) (27) (poloxamer 188) was a gift from BASF ChemTrade GmbH (Burgbernheim, Germany). Miglyol 812 N, caprylic/ capric triglycerides (miglyol) was a gift of Cremer Oleo Division (Witten, Germany). Glyceryl distearate (precirol ATO5, precirol), glyceryl dibehenate (compritol 888ATO, compritol) and mono-, di-, tri-glyceride esters of fatty acids $\left(\mathrm{C}_{10}-\mathrm{C}_{18}\right)$ (suppocire AM, suppocire) were kind gifts of Gattefossè
(Milan, Italy). Glyceryl tristearate (tristearin), $\alpha$-tocopherol (TOC), retinoic acid (RA) and HPLC solvents were purchased from Sigma-Aldrich, Merck (Darmstadt, Germany).

\section{Preparation of lipid nanoparticles}

Lipid nanoparticles were prepared by a hot homogenization technique based on ultrasound treatment. In both cases the dispersing phase was an aqueous solution of poloxamer 188 $(2.5 \% \mathrm{w} / \mathrm{w})$ [37]. In the case of SLN the disperse phase was constituted of one solid lipid (i.e., tristearin, precirol, compritol or suppocire), while in the case of NLC, a mixture between one solid lipid and the liquid lipid caprylic/capric triglycerides (miglyol) (1:1 w/w ratio) was employed. In both cases the lipid phase was 5 or $10 \%$ by weight, with respect to the whole weight of the dispersion. The nanoparticle dispersion acronyms and compositions are reported in Table 1 and Table 2.

Firstly, an emulsion was obtained adding the poloxamer 188 aqueous phase $(4.5 / 4.75 \mathrm{~mL})$ heated at $80{ }^{\circ} \mathrm{C}$ to the molten lipid phase $(250 / 500 \mathrm{mg})$, followed by mixing at $15000 \mathrm{rpm}$, at $80^{\circ} \mathrm{C}$ for $1 \mathrm{~min}$ (IKA T25 digital ultraturrax). Secondly, the emulsion was subjected to ultrasound homogenization at $6.75 \mathrm{kHz}$ for 15 min (Microson ultrasonic Cell Disruptor-XL Minisonix) and

\begin{tabular}{|c|c|c|c|c|c|c|}
\hline \multirow[t]{3}{*}{ preparation } & \multicolumn{6}{|c|}{ composition \% (w/w) } \\
\hline & \multicolumn{4}{|c|}{ lipid phase } & \multicolumn{2}{|c|}{ water phase } \\
\hline & tristearin & compritol & precirol & suppocire & poloxamer & water \\
\hline SLN T5 & 5 & - & - & - & 2.37 & 92.63 \\
\hline SLN T10 & 10 & - & - & - & 2.25 & 87.75 \\
\hline SLN C5 & - & 5 & - & - & 2.37 & 92.63 \\
\hline SLN C10 & - & 10 & - & - & 2.25 & 87.75 \\
\hline SLN P5 & - & - & 5 & - & 2.37 & 92.63 \\
\hline SLN P10 & - & - & 10 & - & 2.25 & 87.75 \\
\hline SLN S5 & - & - & - & 5 & 2.37 & 92.63 \\
\hline SLN S10 & - & - & - & 10 & 2.25 & 87.75 \\
\hline
\end{tabular}

\begin{tabular}{|c|c|c|c|c|c|c|c|}
\hline \multirow[t]{3}{*}{ preparation } & \multicolumn{7}{|c|}{ composition \% (w/w) } \\
\hline & \multicolumn{5}{|c|}{ lipid phase } & \multicolumn{2}{|c|}{ water phase } \\
\hline & tristearin & compritol & precirol & suppocire & miglyol & poloxamer & water \\
\hline NLC T5 & 2.5 & - & - & - & 2.5 & 2.37 & 92.63 \\
\hline NLC T10 & 5.0 & - & - & - & 5.0 & 2.25 & 87.75 \\
\hline NLC C5 & - & 2.5 & - & - & 2.5 & 2.37 & 92.63 \\
\hline NLC C10 & - & 5.0 & - & - & 5.0 & 2.25 & 87.75 \\
\hline NLC P5 & - & - & 2.5 & - & 2.5 & 2.37 & 92.63 \\
\hline NLC P10 & - & - & 5.0 & - & 5.0 & 2.25 & 87.75 \\
\hline NLC S5 & - & - & - & 2.5 & 2.5 & 2.37 & 92.63 \\
\hline NLC S10 & - & - & - & 5.0 & 5.0 & 2.25 & 87.75 \\
\hline
\end{tabular}


allowed to cool at $25^{\circ} \mathrm{C}$. Lipid nanoparticle dispersions were stored at room temperature. In the case of drug-loaded nanoparticles, TOC $(0.4-0.8 \% \mathrm{w} / \mathrm{w}$ with respect to the whole dispersion; $8 \% \mathrm{w} / \mathrm{w}$ with respect to the lipid phase) or RA $(0.02 \% \mathrm{w} / \mathrm{w}$ with respect to the whole dispersion; $0.4 \% \mathrm{w} / \mathrm{w}$ with respect to the lipid phase) were solubilized in caprylic/ capric triglycerides (miglyol) and then added to the fused lipid phase before the emulsification step. The nanoparticle acronyms are reported in Table 3.

\section{Photon correlation spectroscopy (PCS) analysis}

Submicrometer particle analysis was performed using a Zetasizer Nano S90 device (Malvern Instruments, Malvern, England) equipped with a $5 \mathrm{~mW}$ helium neon laser with a wavelength output of $633 \mathrm{~nm}$. The glassware was cleaned of dust by washing with detergent and rinsing twice with water for injections. The measurements were made in triplicate at $25{ }^{\circ} \mathrm{C}$ at an angle of $90^{\circ}$, and the data were interpreted using the "CONTIN" method [38].

\section{Cryogenic transmission electron microscopy (cryo-TEM) analysis}

The samples were vitrified as previously described [39]. The vitrified specimen was transferred to a Zeiss EM922 Omega transmission electron microscope for imaging using a cryoholder (CT3500, Gatan). The temperature of the sample was kept below $-175{ }^{\circ} \mathrm{C}$ throughout the examination. The specimens were examined with doses of about $1000-2000 \mathrm{e} / \mathrm{nm}^{2}$ at $200 \mathrm{kV}$. The images were digitally recorded by a CCD camera (Ultrascan 1000, Gatan) using an image processing system (GMS 1.9 software, Gatan). In addition, the size distribution of the nanoparticles was performed by measuring 1000 nanoparticles for each cryo-TEM image by the digital analyzer ImageJ $1.48 \mathrm{v}$.

\section{Small-angle X-ray scattering (SAXS) mea- surements}

Small-angle X-ray scattering (SAXS) experiments were performed at the SAXS BM29 beamline of the European Synchrotron (ESRF) in Grenoble, France. NLC samples were filled in glass capillaries. The experiments were performed at 30 and $37{ }^{\circ} \mathrm{C}$, both in the presence and absence of TOC and RA. The investigated $Q$-range $(Q=4 \pi \sin \theta / \lambda$, where $2 \theta$ is the scattering angle and $\lambda$ is the $X$-ray wavelength) was from 0.01 to $0.5 \AA^{-1}$, the wavelength used was $0.99 \AA$. The sample exposure time was $160 \mathrm{~s}$, which ensured enough statistical accuracy without degrading the samples by radiation. The Bragg peaks observed were indexed considering the possible symmetries commonly observed in lipid systems (lamellar, hexagonal or cubic) [40]. Accordingly, from the averaged spacing of the observed peaks the unit cell dimension of the phase was calculated.

\section{Encapsulation efficiency and loading capacity of lipid nanoparticles}

The encapsulation efficiency (EE) and loading capacity (LC) of TOC and RA in NLCs were determined as previously described [41]. A $0.5 \mathrm{~mL}$ aliquot of each NLC batch was loaded into a centrifugal filter (Microcon centrifugal filter unit YM-10 membrane, NMWCO 10 kDa, Sigma-Aldrich, St. Louis, MO, USA) and centrifuged (Spectrafuge ${ }^{\text {TM }}$ 24D Digital Microcentrifuge, Woodbridge, NJ, USA) at 8,000 rpm for $20 \mathrm{~min}$. The amount of drug was determined after dissolving the lipid phase with a known amount of methanol $(1: 10 \mathrm{v} / \mathrm{v})$ for $2 \mathrm{~h}$ under stirring. The TOC and RA content was analyzed after filtration by high-performance liquid chromatography (HPLC) using a Knauer Eurospher II RP C18 column (Knauer, Germany) $(15 \times 0.4 \mathrm{~cm})$ stainless steel packed with $5 \mu \mathrm{m}$ particles, eluted at room temperature with different mobile phases. Samples of $50 \mu \mathrm{L}$ were injected through the rheodyne injector system fitted with a $50 \mu \mathrm{L}$ fixed loop and compared with standards of known

\begin{tabular}{|c|c|c|c|c|c|c|c|c|c|}
\hline \multirow[t]{3}{*}{ preparation } & \multicolumn{9}{|c|}{ composition \% (w/w) } \\
\hline & \multicolumn{7}{|c|}{ lipid phase } & \multicolumn{2}{|c|}{ water phase } \\
\hline & tristearin & compritol & precirol & suppocire & miglyol & TOCa & $\mathrm{RA}{ }^{\mathrm{b}}$ & poloxamer & water \\
\hline NLC T5-TOC & 2.5 & - & - & - & 2.5 & 0.4 & - & 2.37 & 92.23 \\
\hline NLC T10-TOC & 5.0 & - & - & - & 5.0 & 0.8 & - & 2.25 & 86.95 \\
\hline NLC C5-TOC & - & 2.5 & - & - & 2.5 & 0.4 & - & 2.37 & 92.23 \\
\hline NLC C10-TOC & - & 5.0 & - & - & 5.0 & 0.8 & - & 2.25 & 86.95 \\
\hline NLC P5-TOC & - & - & 2.5 & - & 2.5 & 0.4 & - & 2.37 & 92.23 \\
\hline NLC P10-TOC & - & - & 5.0 & - & 5.0 & 0.8 & - & 2.25 & 86.95 \\
\hline NLC S5-TOC & - & - & - & 2.5 & 2.5 & 0.4 & - & 2.37 & 92.23 \\
\hline NLC S10-TOC & - & - & - & 5.0 & 5.0 & 0.8 & - & 2.25 & 86.95 \\
\hline NLC T10-RA & 5.0 & - & - & - & 4.98 & - & 0.02 & 2.25 & 87.75 \\
\hline
\end{tabular}

aTOC: $\alpha$-tocopherol; ${ }^{\mathrm{b}} \mathrm{RA}$ : retinoic acid. 
concentration. In the case of TOC, the mobile phase was methanol, and the flow rate was $1 \mathrm{~mL} / \mathrm{min}$ at $295 \mathrm{~nm}$, while for RA, acetonitrile/methanol/methylene chloride $(70: 15: 15, \mathrm{v} / \mathrm{v})$ was employed, with a flow rate of $1 \mathrm{~mL} / \mathrm{min}$ at $325 \mathrm{~nm}$. The analyses were conducted in triplicate. $\mathrm{EE}$ and LC were determined using Equation 1 and Equation 2

$$
\begin{gathered}
\mathrm{EE}=L / T \times 100 \\
\mathrm{LC}=L / T_{\text {lipid phase }} \times 100
\end{gathered}
$$

where $L$ is the amount of drug effectively present within the nanoparticles, $T$ stands for the total amount of drug initially added to the lipid phase and $T_{\text {lipid phase }}$ is the total weight of lipid phase in the formulation. Determinations were performed six times in independent experiments and the mean values \pm standard deviations were calculated.

\section{Stability studies}

After production, the nanoparticles were stored in glass containers at $25{ }^{\circ} \mathrm{C}$ for 6 months [42]. To assess the physical and chemical stability, particle size analysis and TOC encapsulation efficiency were periodically evaluated by PCS and HPLC, respectively, as above reported.

\section{Western blot analysis for $\mathrm{HO}-1$ and $\mathrm{HO}-2$ protein \\ Cytotoxicity determination}

Experiments were carried out to assess the range of NLC T10TOC, NLC C10-TOC, NLC P10-TOC and NLC S10-TOC concentrations that are nontoxic for cells. Briefly, human immortalized keratinocytes (HaCaT) were treated for $24 \mathrm{~h}$ with the different NLC formulations at various TOC concentrations, ranging from 25 to $200 \mu \mathrm{M}$. Cytotoxicity was evaluated by spectrophotometric quantification of the LDH released in culture medium, using a commercial kit (Sigma-Aldrich, Merck, Darmstadt, Germany), as previously described [43].

\section{Human skin explant (HSE) culture}

Skin explants were prepared from the superfluous skin of healthy adult donors (18-60 years old). Breast or abdominal tissue specimens were obtained from patients undergoing plastic surgery. Skin biopsies (12 mm punches) were cultured into standard 6-well plates in contact with culture medium at $37{ }^{\circ} \mathrm{C}$ in $5 \% \mathrm{CO}_{2}$ humidified air. The culture medium was Dulbecco's Modified Eagle Medium (DMEM) with 1\% antibiotic-antimycotic solution $(10,000$ units penicillin, $10 \mathrm{mg}$ streptomycin and $25 \mu \mathrm{g}$ amphotericin B - Sigma-Aldrich, Germany) and 1\% L-glutamine (Sigma-Aldrich, Germany) [43]. After 1 day in culture, the medium was changed and HSEs were topically treated with $50 \mu \mathrm{L}$ of NLC T10 and NLC T10-TOC for $24 \mathrm{~h}$.

\section{Cigarette smoke (CS) exposure}

After $24 \mathrm{~h}$ of treatment, the HSEs were exposed for 30 minutes to CS generated by burning one research cigarette $(12 \mathrm{mg}$ tar, $1.1 \mathrm{mg}$ nicotine) using a vacuum pump, as previously described [44]. Control HSEs were exposed to filtered air. After exposure, the explants were incubated in fresh media at $37{ }^{\circ} \mathrm{C}$ in a humidified $5 \% \mathrm{CO}_{2}$ atmosphere for $24 \mathrm{~h}$.

\section{Protein extraction}

Samples for Western blot analysis were washed in PBS and frozen in liquid nitrogen. The biopsies were extracted in icecold conditions using a tissue protein extraction reagent (T-PER buffer, Thermo Fisher Scientific, MA, USA) added consisting of protease and phosphatase inhibitor cocktails (Sigma, Milan, Italy), using a bead-based homogenizer at $12400 \mathrm{rpm}$ at $4{ }^{\circ} \mathrm{C}$ for $15 \mathrm{~min}$. The protein concentration was measured by the Bradford method (BioRad, CA, USA) [40].

\section{Western blot analysis}

The samples ( $25 \mu \mathrm{g}$ protein) were loaded onto $10 \%$ sodium dodecyl sulfate polyacrylamide gel (SDS-PAGE) and then transferred onto nitrocellulose membranes. Blots were blocked in PBS containing $0.5 \%$ Tween 20 and 5\% not-fat milk (BioRad). The membranes were incubated overnight at $4{ }^{\circ} \mathrm{C}$ with the appropriate primary antibody HO-1 (Abcam, Cambridge, UK). The membranes were then incubated with horseradish peroxidase conjugated secondary antibody for $1 \mathrm{~h}$ at RT, and the bound antibodies were detected in a chemiluminescent reaction (ECL, BioRad). Chemiluminescence was detected on a ChemiDoc imager (BioRad) [45]. The blots were reprobed with $\beta$-actin as the loading control. Images of the bands were digitized, and the densitometry of the bands was performed using ImageJ software [46].

\section{Statistical analysis}

For each of the variables tested, two-way analysis of variance (ANOVA) was used. A significant result was indicated by a $p$ value $<0.05$. All the results are expressed as mean $\pm \mathrm{SD}$ of 6 determinations for nanoparticle characterization experiments and 3 determinations obtained in 3 independent experiments for in vitro cultured cells tests.

\section{Results and Discussion Effect of lipid composition on nanoparticle macrostructure}

In order to obtain a nanoparticulate system suitable for cutaneous administration of antioxidants, different lipid compositions have been considered, as reported in Table 1 and Table 2. 
The selection of the lipid composition has been performed by choosing nottoxic, commercial lipids with similar chemical composition and different carbon chain lengths on the basis of our expertise concerning tristearin and caprylic/capric triglycerides (miglyol) [39]. Namely, SLNs were produced based on the use of solid di- or tri-glycerides, with chain lengths ranging between 18 and 21 carbon atoms, while for NLC production, the same solid lipids were employed in mixture $(1: 1 \mathrm{w} / \mathrm{w})$ with the liquid caprylic/capric triglycerides (miglyol), characterized by $\mathrm{C}_{8}-\mathrm{C}_{10}$ chains.

With regard to surfactant concentration, higher poloxamer amounts, namely 3 and $4 \% \mathrm{w} / \mathrm{w}$ with respect to the aqueous phase, have been tested. However, the increase of poloxamer led to foam formation during the preparation, which caused irregular and inhomogeneous formulations; thus $2.5 \% \mathrm{w} / \mathrm{w}$ of poloxamer was used.

The hot homogenization method followed by ultrasound [39] lead to production of milky and homogeneous dispersions. Immediately after cooling, in most cases, a small amount of coalesced lipid phase appeared on the surface of the dispersion. The weight of this agglomerate, spanning between 0 and $4.65 \%$ by weight with respect to the total amount of the lipid phase, was a function of the lipid composition. Indeed, both the amount and the type of lipid phase appear to influence the agglomerate formation. Particularly, the longer the lipid chain, the higher the agglomerate weight according to the following trend: compritol $\left(2 \mathrm{C}_{21}\right.$ chains $)>$ tristearin $\left(3 \mathrm{C}_{18}\right.$ chains $)>$ precirol $\left(2 \mathrm{C}_{18}\right.$ chains $)>$ suppocire $\left(3 \mathrm{C}_{10}-\mathrm{C}_{18}\right.$ chains $)$. Specifically, in the latter case, the agglomerate was almost absent (Table 4). In addition, the extent of agglomeration was lower for NLC, probably due to the presence of the liquid lipid.

\section{Effect of lipid composition on nanoparticle size distribution}

The SLN and NLC dimensions, measured by PCS and expressed by the Z-average, $D_{\mathrm{Z}}$, are reported in Figure 1 and Table 4 . In the case of SLN with 5\% lipid phase concentration, mean diameters were comprised between 148 and $245 \mathrm{~nm}$, with some differences due to the lipid composition. The doubling of the lipid phase concentration, however, induced an increase of the mean diameter, especially in the case of SLN P10 and SLN $\mathrm{C} 10$, whose $\mathrm{Z}$-average reached almost $500 \mathrm{~nm}$. In the case of NLC, the lipid phase composition scarcely affected the mean diameter, ranging between 125 and $160 \mathrm{~nm}$ both for $5 \%$ and $10 \%$ of lipid phase. The polydispersity index was always below 0.39 , with smaller values in the case of NLCs.

The Z-average mean diameters of SLNs and NLCs stored at $25{ }^{\circ} \mathrm{C}$ were measured after 3 months from production. In the
Table 4: Dimensional characteristics of SLNs or NLCs and the presence of agglomerates.

\begin{tabular}{llll} 
Preparation & $\begin{array}{l}\text { Z-average, } \\
D_{z}(\mathrm{~nm})\end{array}$ & $\begin{array}{l}\text { Polydispersity } \\
\text { index }\end{array}$ & $\begin{array}{l}\text { Agglomerate } \\
(\%)\end{array}$ \\
\hline SLN T5 & $148.6 \pm 74.5$ & $0.35 \pm 0.11$ & $2.86 \pm 0.04$ \\
SLN T10 & $164.9 \pm 9.4$ & $0.33 \pm 0.04$ & $4.65 \pm 0.03$ \\
SLN C5 & $244.5 \pm 26.5$ & $0.36 \pm 0.01$ & $3.43 \pm 0.02$ \\
SLN C10 & $488.9 \pm 25.3$ & $0.32 \pm 0.04$ & $3.77 \pm 0.04$ \\
SLN P5 & $245.5 \pm 31.9$ & $0.31 \pm 0.03$ & $2.48 \pm 0.03$ \\
SLN P10 & $453.1 \pm 9.3$ & $0.39 \pm 0.02$ & $2.99 \pm 0.04$ \\
SLN S5 & $220.9 \pm 15.6$ & $0.37 \pm 0.06$ & $1.13 \pm 0.03$ \\
SLN S10 & $201.8 \pm 82.1$ & $0.39 \pm 0.06$ & $0.60 \pm 0.04$ \\
\hline NLC T5 & $122.6 \pm 34.2$ & $0.32 \pm 0.07$ & $2.14 \pm 0.02$ \\
NLC T10 & $127.9 \pm 29.1$ & $0.29 \pm 0.03$ & $2.54 \pm 0.01$ \\
NLC C5 & $160.2 \pm 25.5$ & $0.29 \pm 0.03$ & $3.15 \pm 0.02$ \\
NLC C10 & $136.8 \pm 51.5$ & $0.24 \pm 0.04$ & $3.25 \pm 0.01$ \\
NLC P5 & $148.1 \pm 29.5$ & $0.22 \pm 0.10$ & $1.51 \pm 0.01$ \\
NLC P10 & $159.8 \pm 33.7$ & $0.30 \pm 0.08$ & $1.84 \pm 0.02$ \\
NLC S5 & $131.3 \pm 30.5$ & $0.31 \pm 0.05$ & $0 \pm 0.01$ \\
NLC S10 & $136.1 \pm 13.5$ & $0.29 \pm 0.07$ & $0 \pm 0.01$ \\
\hline
\end{tabular}

aLoss of lipids (lipid phase) due to the partial coalescence of the lipid phase during the formation of the $\mathrm{O} / \mathrm{W}$ emulsion. \% refers to the weight of the lipid phase. Data represent the mean \pm SD of 6 independent experiments.

case of SLNs, the mean diameter dramatically increased, as reported in Figure 1A and 1B, especially for SLN T10, SLN C10 and SLN P10, reaching values undetectable by PCS, where instead, the mean diameter of SLN S5 and SLN S10 did not improve. This behavior can be attributed to the lipid phase containing SLN that influences both agglomerate and the mean diameter of the nanoparticles. On the contrary, the NLCs maintained their mean diameters almost unvaried, irrespectively of the lipid phase type and concentration (Figure 1C and 1D).

In order to avoid agglomeration phenomena and to control the mean size, only NLCs have been considered for antioxidant loading.

\section{Production and characterization of NLCs containing antioxidants}

To produce antioxidant-containing NLCs, different amounts of TOC and RA were loaded in NLCs, as reported in Table 3. Particularly, since TOC is practically insoluble in water ( $\log \mathrm{P} 8.84)$, it was directly added to caprylic/capric triglycerides (miglyol) in order to improve its solubility (reaching $16 \mathrm{mg} / \mathrm{mL}$ ) before addition of solid lipids [47]. The doubling of the lipid phase concentration enabled to doubling the amount of TOC loading. The macroscopic characteristic of NLCs containing TOC was milky and homogeneous, similar to the empty NLCs. Both the presence of agglomerates and the mean size of 

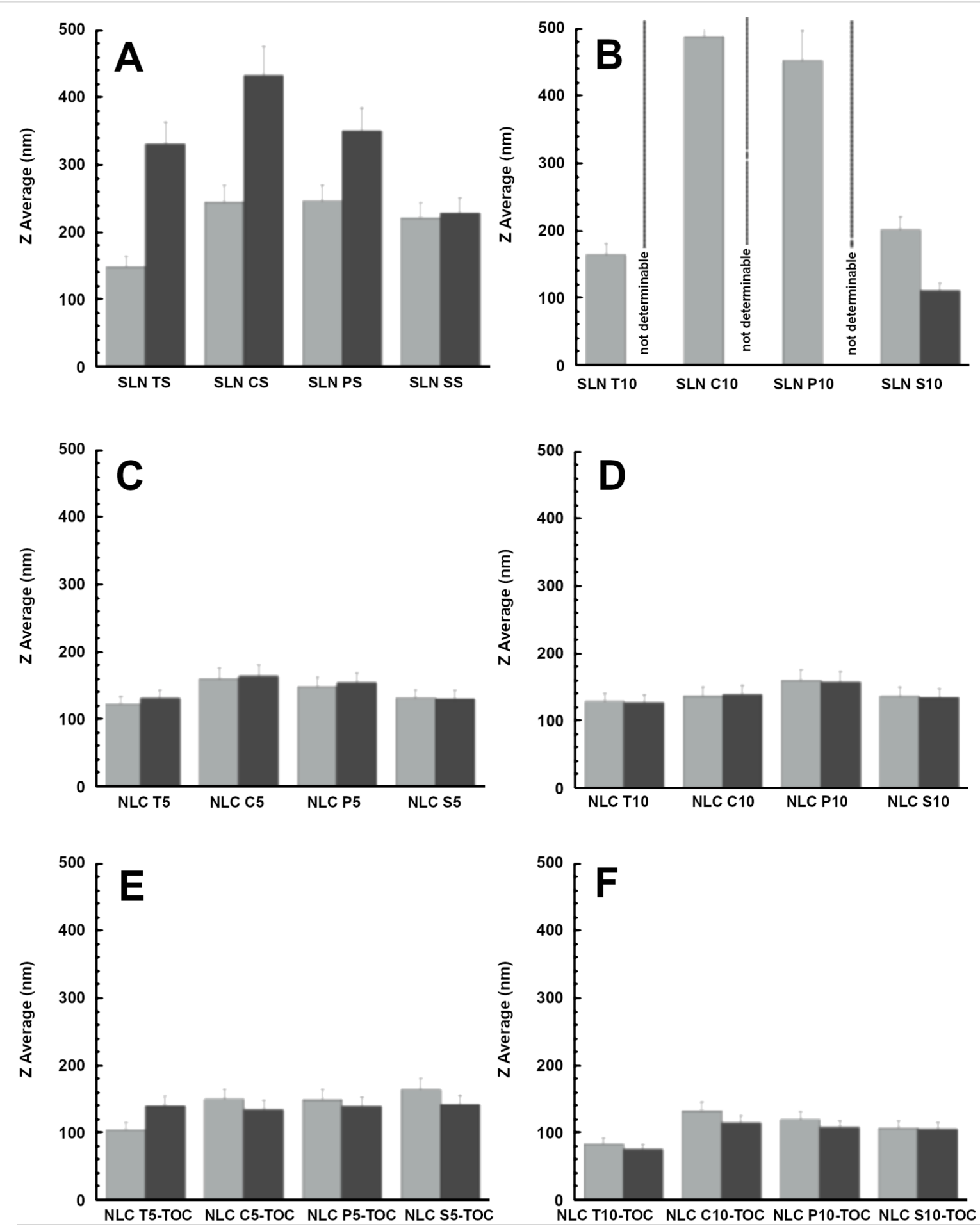

Figure 1: Variation of the Z-average mean diameters, $D_{Z}$, of SLNs (A, B) and NLCs (C, D) produced in the absence of antioxidants and NLC loaded with TOC (E, F) evaluated at 1 (light grey) and 90 (grey) days after nanoparticle production. In the case of SLN T10, SLN C10 and SLN P10, mean diameters were not measurable by PCS 90 days after production.

NLCs containing TOC were lower with respect to their empty counterparts (Table 5). This trend suggest that TOC could contribute to stabilize the interface between the lipid and the aqueous phase, leading to smaller droplets and finally to smaller nanoparticles. The agglomerate presence was more evident in the case of compritol and absent in the case of suppocire, as in the case of empty NLCs, while mean dimensions were inversely proportional to the amount of lipid phase and TOC. As ob- 


\begin{tabular}{|c|c|c|c|c|c|}
\hline NLC preparation & Z-average, $D_{\mathrm{z}}(\mathrm{nm})$ & Polydispersity index & Agglomerate $^{\mathrm{a}}(\%)$ & Encapsulation efficiency ${ }^{b}$ & Loading capacity ${ }^{\mathrm{C}}$ \\
\hline NLC T5-TOC & $104.5 \pm 32.0$ & $0.33 \pm 0.11$ & $1.22 \pm 0.02$ & $90.96 \pm 1.3$ & $7.27 \pm 0.1$ \\
\hline NLC T10-TOC & $82.8 \pm 10.7$ & $0.36 \pm 0.05$ & $1.24 \pm 0.01$ & $90.69 \pm 2.8$ & $7.25 \pm 0.2$ \\
\hline NLC C5-TOC & $149.4 \pm 36.9$ & $0.22 \pm 0.03$ & $2.25 \pm 0.01$ & $95.61 \pm 1.5$ & $7.64 \pm 0.2$ \\
\hline NLC C10-TOC & $132.7 \pm 51.3$ & $0.34 \pm 0.05$ & $3.17 \pm 0.02$ & $79.15 \pm 2.5$ & $6.33 \pm 0.1$ \\
\hline NLC P5-TOC & $149.5 \pm 30.3$ & $0.30 \pm 0.02$ & $1.11 \pm 0.01$ & $93.58 \pm 1.7$ & $7.48 \pm 0.1$ \\
\hline NLC P10-TOC & $118.5 \pm 31.9$ & $0.30 \pm 0.05$ & $1.12 \pm 0.02$ & $90.99 \pm 2.2$ & $7.27 \pm 0.2$ \\
\hline NLC S5-TOC & $164.6 \pm 21.7$ & $0.29 \pm 0.06$ & $0.00 \pm 0.01$ & $88.16 \pm 1.3$ & $7.05 \pm 0.1$ \\
\hline NLC S10-TOC & $106.1 \pm 24.2$ & $0.29 \pm 0.07$ & $0.00 \pm 0.01$ & $60.72 \pm 2.1$ & $4.85 \pm 0.1$ \\
\hline NLC T10-RA & $98.4 \pm 20.2$ & $0.27 \pm 0.12$ & $2.52 \pm 0.01$ & $67.24 \pm 0.8$ & $0.16 \pm 0.0$ \\
\hline
\end{tabular}

aLoss of lipids (lipid phase) due to the partial coalescence of the lipid phase during the formation of the O/W emulsion. After cooling the coalesced lipid phase appeared as a small flake floating on the surface of the NLC dispersion. bercentage (w/w) of drug in the whole dispersion with respect to the total amount used for the preparation. ${ }^{\mathrm{c} P e r c e n t a g e}(\mathrm{w} / \mathrm{w})$ of drug within nanoparticles as compared to the amount of lipid used for the preparation. Data represent the mean \pm S.D. of 6 independent experiments.

served in the case of the empty counterparts, Z-average mean diameters, $D_{\mathrm{Z}}$, of antioxidants containing NLC stored at $25^{\circ} \mathrm{C}$ for 3 months were almost unvaried (Figure $1 \mathrm{E}$ and $1 \mathrm{~F}$ ). Particularly, NLC T10-TOC displayed the smallest mean diameter, even after 3 months.

Due to the encouraging results obtained using tristearin $10 \%$, RA was loaded into NLC T10. Due to its poor water solubility ( $\log$ P 6.3), as in the case of TOC, RA was added to caprylic/ capric triglycerides (miglyol), improving its solubility to $4 \mathrm{mg} / \mathrm{mL}$ [48]. In the case of NLC T10-RA, despite the small mean diameter $(98 \mathrm{~nm})$, the agglomeration phenomenon was more noticeable as compared to NLC T10-TOC (Table 5).

The NLC morphology was investigated by cryo-TEM and a few images are reported in Figure 2. In general, the NLC shape appears discoid in the top view, or more electron-dense and rodlike in the edge-on view. In the case of tristearin-based NLCs, the shape was roundish, both for empty (Figure 2A) and antioxidant-loaded NLC T10 (Figure 2B and C). In the case of compritol (Figure 2D) and precirol (Figure 2E) based NLCs, ovoid and triangular structures were observed. At last, in the case of suppocire NLCs, besides the presence of some irregular structures (Figure 2F), spherical structures were detected (inset of Figure $2 \mathrm{~F}$ ), resembling vesicles rather than to solid particles.

The inner morphology of the NLCs was further characterized by SAXS [39,40]. In particular, SAXS experiments were performed on NLC samples prepared by using tristearin or suppocire both in the presence and in the absence of TOC and RA. By way of illustration, Figure 3 shows the low-angle diffraction profiles obtained as a function of the lipid phase concentration from tristearin-based NLCs (top graph, A) and from suppocire-based NLCs (middle graph, B), both containing
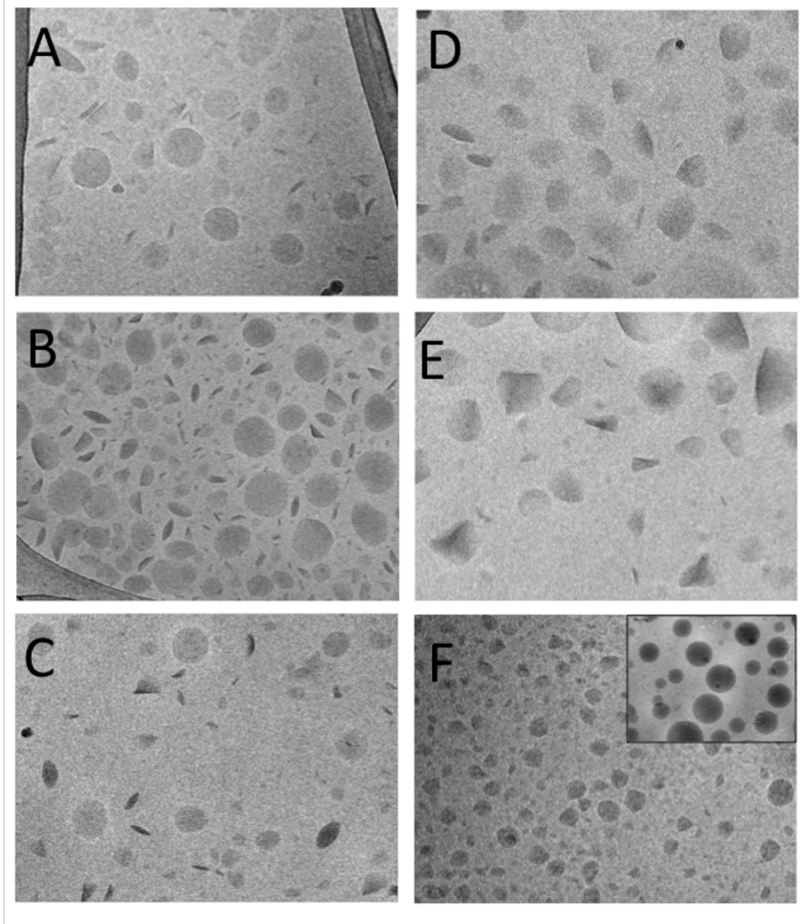

Figure 2: Cryo-TEM images of NLC T10 (A), NLC T10-TOC (B), NLC T10-RA (C), NLC C5-TOC (D), NLC P5-TOC (E), NLC S5-TOC (F). The scale bar below corresponds to $200 \mathrm{~nm}$ in panels $A-E$ and $300 \mathrm{~nm}$ in panel $\mathrm{F}$.

TOC. The presence of Bragg peaks in the NLC T5-TOC and NLC T10-TOC samples shows that the inner structure of the $\mathrm{NLC}$ at $30{ }^{\circ} \mathrm{C}$ depends on the used lipid: tristearin guarantees the presence of an ordered structural organization inside the NLC, while suppocire is not able to preserve such an organization. According to the cryo-TEM findings, vesicles rather than nanoparticles probably form in this condition. 

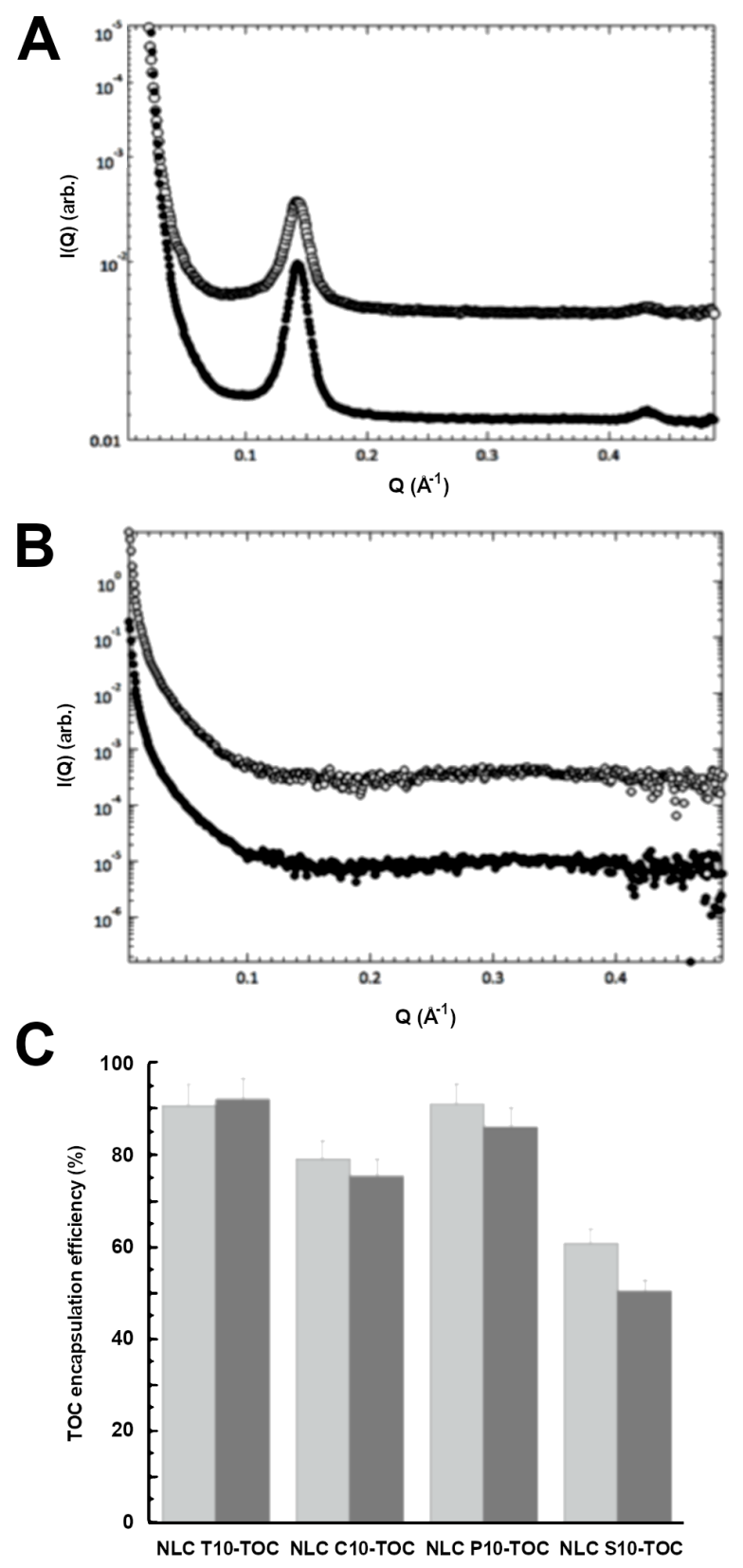

Figure 3: SAXS profiles observed for A: NLCT5-TOC (open symbol) and NLCT10-TOC (closed symbol), B: NLCS5-TOC (open symbol), and NLCS10-TOC (closed symbol). C: TOC encapsulation efficiency in the indicated NLC, evaluated at 1 (light grey) and 90 (grey) days after production.

The analysis of the position of the peaks observed in tristearinbased NLCs allowed the identification of the internal structural organization for NLC T5-TOC and NLC T10-TOC: because the spacing ratios scale as 1:2:3.., a lamellar organization was derived [34,35]. The corresponding unit cell dimension, which in a lamellar organization is the repeated distance between two lamellae, was $43.9 \AA$ A. It should be noted that similar results were obtained from empty tristearin-based NLCs: the packing of the lamellae in the nanoparticle inner region is not affected by the presence of the antioxidant. The scattering profiles obtained from NLC T5-RA and NLC T10-RA were very similar (data not shown), confirming the described behavior.

\section{Encapsulation of antioxidants in NLCs}

The influence of the NLC lipid composition on the capability to incorporate antioxidants was studied by evaluating the EE and LC. The values reported in Table 5 and Figure $3 \mathrm{C}$ evidenced that in the case of tristearin or precirol based NLCs, the EE of TOC was $>90 \%$, irrespective of the lipid phase concentration. In the case of NLC C10-TOC, both a decrease in the EE value and an increase in agglomeration were detected with respect to NLC C5-TOC, suggesting that the doubling of the lipid concentration promoted agglomeration of the lipid phase, partially avoiding TOC encapsulation within the nanoparticles. This hypothesis was corroborated by disaggregation and HPLC analysis of the lipid phase agglomerate, revealing the presence of $13 \% \mathrm{w} / \mathrm{w}$ TOC with respect to the total amount used for NLC production.

The lowest EE values were found in the case of NLC S10-TOC and NLC T10-RA. In the case of suppocire, TOC EE values decreased from 88 to $60 \%$ by doubling the lipid phase concentration, suggesting that the presence of vesicles instead of nanoparticles prevented high loading of the antioxidant within their structure.

Regarding NLC T10-RA, as for NLC C10-TOC, an amount of antioxidant (11\%) was found within the agglomerate of the lipid phase, justifying the reason for the low EE value of RA. LC values of NLCs containing TOC were between 4.85 and $7.64 \%$, whilst in the case of RA, the LC was only $0.16 \%$ due to the lower amount of RA employed for NLC production $(0.05 \mathrm{mg} / 100 \mathrm{mg}$ lipid phase, instead of $8 \mathrm{mg} / 100 \mathrm{mg}$ lipid phase used in the case of TOC).

In order to detect the capability of NLC to control the encapsulation of antioxidants under storage, the EE values were evaluated for 90 days (Figure 3C). Particularly, NLCs containing $10 \%$ lipid phase were selected due to their marked dimensional stability.

The TOC EE values were almost unvaried in the case of NLC T10-TOC - they slightly decreased in the case of NLC C10TOC and NLC P10-TOC, whilst the decrease was more evident in the case of NLC S10-TOC, passing from 60 to $48 \%$. It can be hypothesized that the prevalence of vesicles in NLC S10-TOC, instead of more structured carriers, hindered the TOC encapsulation. Lastly, in the case of NLC T10-RA, RA encapsulation dramatically decreased -the EE value halved one month after 
production (data not shown). Due to their poor stability, NLC T10-RA samples were not considered for further studies.

\section{Cytotoxicity of NLCs containing TOC}

As the produced formulations are intended for topical administration on the skin, experiments on human keratinocytes were conducted in order to test the cytotoxicity of NLC T10-TOC, NLC C10-TOC, NLC P10-TOC and NLC S10-TOC. The LDH release in the media was assessed $24 \mathrm{~h}$ after TOC treatment at the concentrations of $25,50,100$ and $200 \mu \mathrm{M}$.

As shown in Figure 4, no NLC cytotoxicity was observed with respect to control cells and no significant difference among the different NLCs was noticed, confirming the biocompatibility of the components.
Due to the obtained results, NLC T10-TOC samples were selected for further ex vivo studies. Indeed, this kind of NLC displayed better physico-chemical properties with respect to NLC based on different lipid compositions, being able to longer maintain the size and the EE of TOC.

\section{Antioxidant effect of NLCs containing TOC}

Following the results obtained in the 2D cell model, the study of the protective effect of NLC T10-TOC was carried out on HSE.

CS contains many components able to elicit oxidative stress, which can induce the cytoprotective enzyme heme oxygenase (HO-1). An HO-1 increase promotes protection against inflammation and/or cell death induced by CS $[49,50]$. In order to

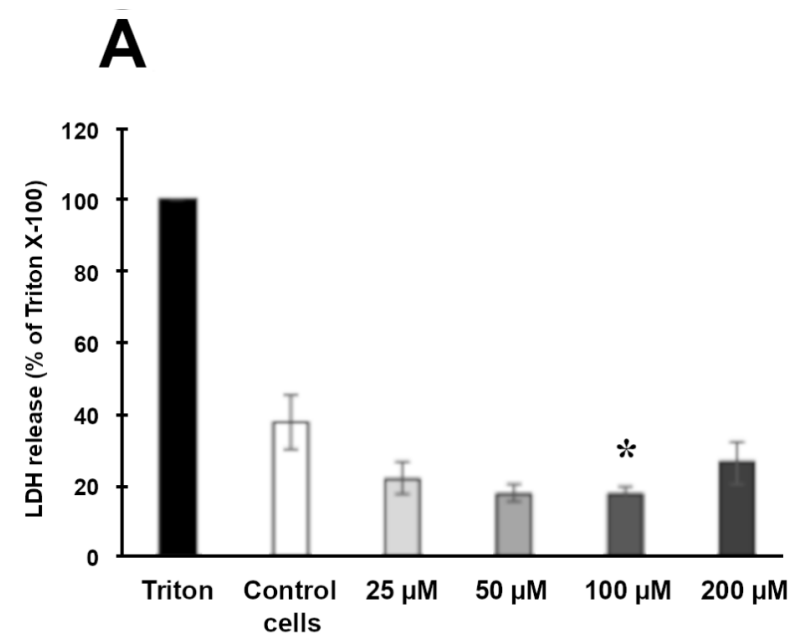

C

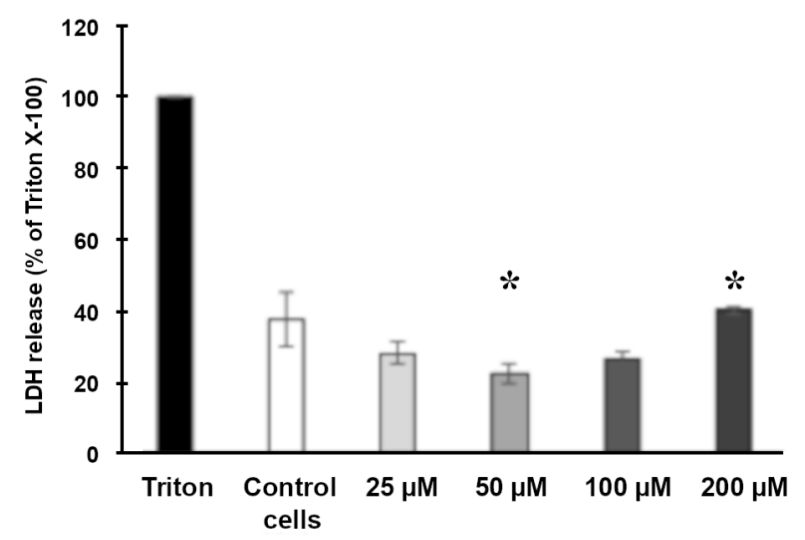

B

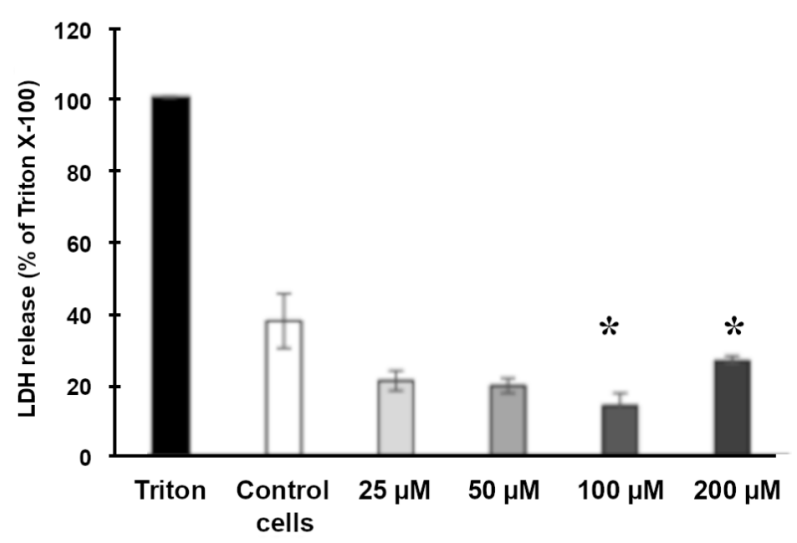

D

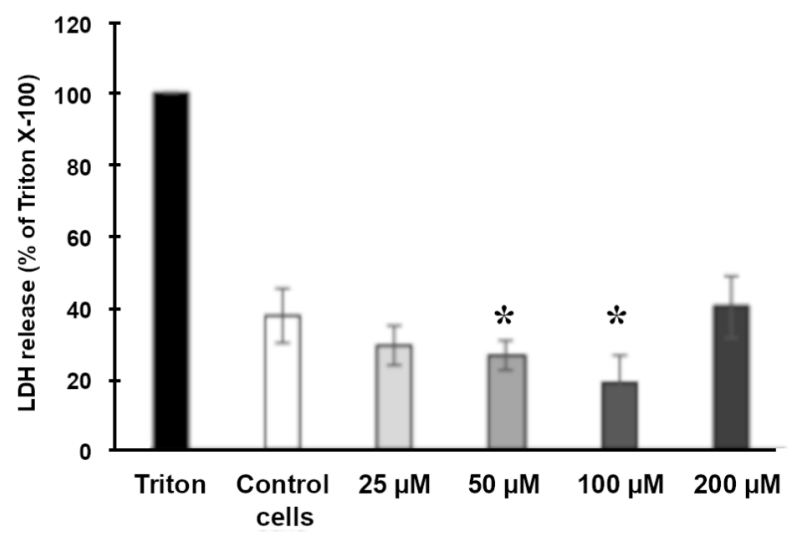

Figure 4: Cytotoxicity of NLC T10-TOC (A), NLC C10-TOC (B), NLC P10-TOC (C) and NLC S10-TOC (D) evaluated by LDH release from HaCaT cells in the media after $24 \mathrm{~h}$ of treatment. Data are expressed as percentage LDH release as compared to the maximum release of LDH from TritonX100-treated cells. Data are given as mean \pm SD, representative of three independent experiments with at least three technical replicates each time. ${ }^{*}$ indicates statistically significant difference to untreated control cells (unpaired $t$-test, $p<0.01$ ). 
evaluate the effect of NLC T10-TOC in preventing damage caused by $\mathrm{CS}$, the HO-1 expression was evaluated on HSE cultures exposed to CS or to air for $24 \mathrm{~h}$. Namely HO-1 has been determined by Western blot analysis, quantified by densitometry and normalized to the beta-actin level for each sample (Figure 5A). The mean relative density ratios of three experiments are shown in Figure 5B. As depicted, the expression of the HO-1 protein level is significantly induced by the CS exposure because of the ability of this outdoor stressor to promote oxidative-related cellular modifications to the skin [39]. On the other hand, HO-1 levels in skin explants treated with NLC T10TOC and exposed to CS were dramatically and significantly prevented (47\% decrease, $p<0.001$ vs control).
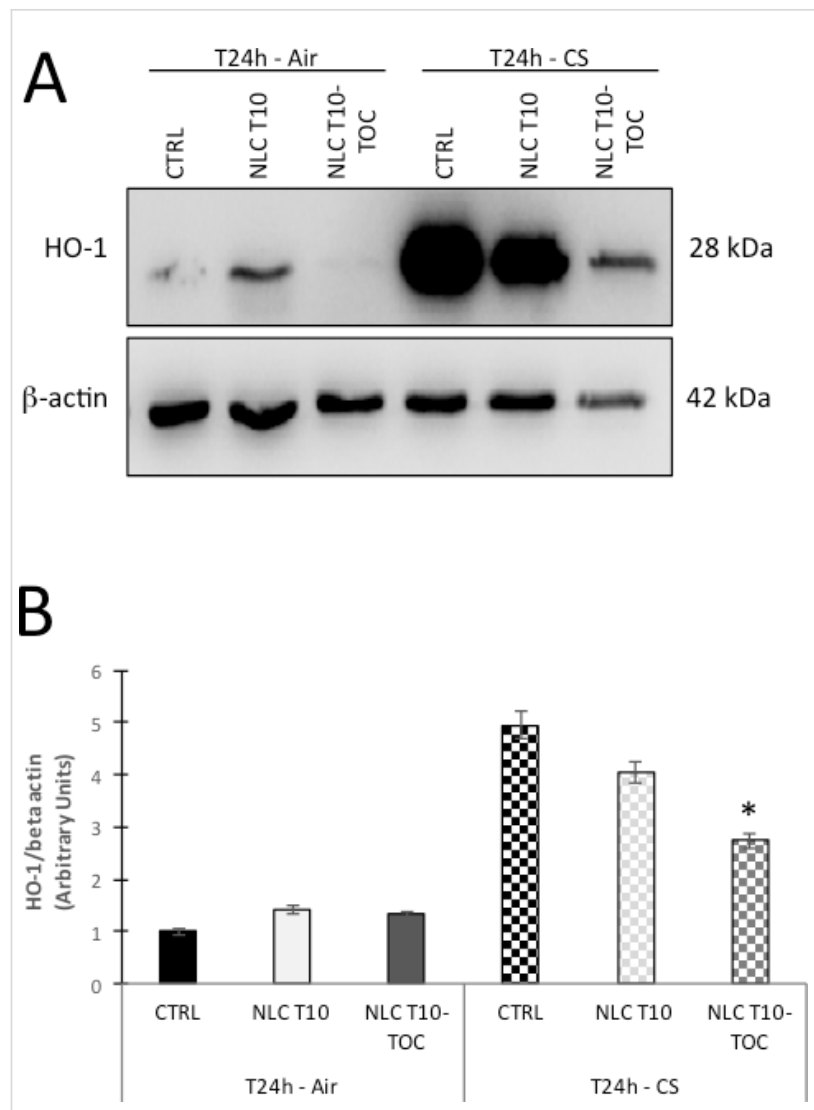

Figure 5: Effect of cigarette smoke (CS) on heme-oxygenase (HO-1) expression evaluated on human skin explants (HSE) treated with NLCT 10 or NLCT 10-TOC, exposed to air or CS for $30 \mathrm{~min}$ and harvested after $24 \mathrm{~h}$. A) Representative Western blot analyses of HO-1 protein expression (with the respective $\beta$-actin controls). B) Mean expression of $\mathrm{HO}-1$ as a ratio of $\beta$-actin. The results are shown as the mean of three experiments. ${ }^{*} p<0.05$ with respect to the control.

These results suggest that NLC T10-TOC can effectively reduce the induction of cutaneous HO-1, which is a sensor of tissue stress, suggesting the ability of this topical application to prevent CS-induced skin damage. Further studies will be required to investigate the dose and type-dependent manner of action of TOC loaded in NLCs with respect to an unloaded TOC solution.

\section{Conclusion}

This work has underlined the importance of technological screening in the design of a nanoparticulate lipid dosage formulation. Notably, dimensional and morphological characterization of nanoparticles should be performed at different durations of time after production. This investigation has demonstrated that the type and concentration of the lipid phase affect the physico-chemical stability of nanoparticles. The NLC T10-TOC sample that was selected by the preformulation study deserved further in vitro and in vivo studies. Indeed, supplementary studies will be performed to investigate the activity of hydrophilic antioxidant molecules, such as ascorbic acid and $\mathrm{N}$-acetyl-cysteine, loaded in NLCs and in comparison with conventional "non-nano" formulations. In addition, since some authors have demonstrated that CS induces depletion of some essential vitamins, such as TOC and RA [23], it should be interesting to evaluate the suitability of NLC T10-TOC as an oral antioxidant supplement.

\section{Acknowledgements}

This work was funded by "FIR 2018" of Ferrara University, Italy.

\section{ORCID ${ }^{\circledR}$ iDs}

Elisabetta Esposito - https://orcid.org/0000-0002-8416-3629 Maddalena Sguizzato - https://orcid.org/0000-0002-2100-2043 Markus Drechsler - https://orcid.org/0000-0001-7192-7821 Paolo Mariani - https://orcid.org/0000-0003-4293-1009 Federica Carducci - https://orcid.org/0000-0002-3985-7998 Rita Cortesi - https://orcid.org/0000-0002-1060-2676

\section{References}

1. Rembiesa, J.; Ruzgas, T.; Engblom, J.; Holefors, A. Cosmetics 2018, 5, 4-12. doi:10.3390/cosmetics5010004

2. Saha, S. P.; Bhalla, D. K.; Whayne, T. F.; Gairola, C. G. Int. J. Angiol. 2007, 16, 77-83. doi:10.1055/s-0031-1278254

3. Onor, I. O.; Stirling, D. L.; Williams, S. R.; Bediako, D.; Borghol, A.; Harris, M. B.; Darensburg, T. B.; Clay, S. D.; Okpechi, S. C.; Sarpong, D. F. Int. J. Environ. Res. Public Health 2017, 14, 1147. doi:10.3390/ijerph14101147

4. Kennedy, C.; Bastiaens, M. T.; Willemze, R.; Bouwes Bavinck, J. N.; Bajdik, C. D.; Westendorp, R. G. J. J. Invest. Dermatol. 2003, 120 , 548-554. doi:10.1046/j.1523-1747.2003.12092.x

5. Silvano, A.; Nistri, S.; Calosi, L.; Romagnoli, P. Ital. J. Anat. Embryol. 2019, 124, 42-57.

6. Suehara, L. Y.; Simone, K.; Maia, M. An. Bras. Dermatol. 2006, 81, 34-39. doi:10.1590/s0365-05962006000100004

7. Hu, S. S.; Neff, L.; Agaku, I. T.; Cox, S.; Day, H. R.; Holder-Hayes, E.; King, B. A. Morb. Mortal. Wkly. Rep. 2016, 65, 685-691. doi:10.15585/mmwr.mm6527a1 
8. Benowitz, N. L.; Hukkanen, J.; Jacob, P., III. Nicotine Chemistry, Metabolism, Kinetics and Biomarkers. In Handbook of Experimental Pharmacology; Barrett, J. E., Ed.; Springer Berlin: Berlin, Germany; pp 29-60. doi:10.1007/978-3-540-69248-5_2

9. Bruno, R. S.; Traber, M. G. J. Nutr. 2005, 135, 671-674. doi:10.1093/jn/135.4.671

10. Bruno, R. S.; Leonard, S. W.; Atkinson, J.; Montine, T. J.; Ramakrishnan, R.; Bray, T. M.; Traber, M. G. Free Radical Biol. Med. 2006, 40, 689-697. doi:10.1016/j.freeradbiomed.2005.10.051

11. Alberg, A. J. Toxicology 2002, 180, 121-137. doi:10.1016/s0300-483x(02)00386-4

12. Thomsen, S. F.; Sørensen, L. T. Skin Therapy Lett. 2010, 15, 4-7.

13. Handelman, G. J.; Packer, L.; Cross, C. E. Am. J. Clin. Nutr. 1996, 63, 559-565. doi:10.1093/ajcn/63.4.559

14. Dietrich, M.; Block, G.; Norkus, E. P.; Hudes, M.; Traber, M. G.; Cross, C. E.; Packer, L. Am. J. Clin. Nutr. 2003, 77, 160-166. doi:10.1093/ajcn/77.1.160

15. Mistry, N. Cosmetics 2017, 4, 57. doi:10.3390/cosmetics4040057

16. Wolf, G. J. Nutr. 2005, 135, 363-366. doi:10.1093/jn/135.3.363

17. Buettner, G. R. Arch. Biochem. Biophys. 1993, 300, 535-543. doi:10.1006/abbi.1993.1074

18. Chen, J.; Wei, N.; Lopez-Garcia, M.; Ambrose, D.; Lee, J.; Annelin, C.; Peterson, T. Eur. J. Pharm. Biopharm. 2017, 117, 286-291. doi:10.1016/j.ejpb.2017.04.008

19. Vaz, S.; Silva, R.; Amaral, M. H.; Martins, E.; Sousa Lobo, J. M.; Silva, A. C. Colloids Surf., B 2019, 179, 242-249. doi:10.1016/j.colsurfb.2019.03.036

20. Okuno, M.; Kojima, S.; Matsushima-Nishiwaki, R.; Tsurumi, H.; Muto, Y.; Friedman, S.; Moriwaki, H. Curr. Cancer Drug Targets 2004, 4, 285-298. doi:10.2174/1568009043333023

21. Ross-Innes, C. S.; Stark, R.; Holmes, K. A.; Schmidt, D.; Spyrou, C.; Russell, R.; Massie, C. E.; Vowler, S. L.; Eldridge, M.; Carroll, J. S. Genes Dev. 2010, 24, 171-182. doi:10.1101/gad.552910

22. Uray, I. P.; Dmitrovsky, E.; Brown, P. H. Semin. Oncol. 2016, 43, 49-64. doi:10.1053/j.seminoncol.2015.09.002

23. Xue, Y.; Harris, E.; Wang, W.; Baybutt, R. C. J. J. Biomed. Sci. (London, U. K.) 2015, 22, 84-92. doi:10.1186/s12929-015-0189-0

24. Andersen, F. A. Int. J. Toxicol. 2002, 21, 51-116. doi:10.1080/10915810290169819

25. MacGregor, J. L.; Maibach, H. I. Exog. Dermatol. 2002, 1, 68-73. doi:10.1159/000058335

26. Saez, V.; Souza, I. D. L.; Mansur, C. R. E. Int. J. Cosmet. Sci. 2018, 40, 103-116. doi:10.1111/ics.12452

27. Eiras, F.; Amaral, M. H.; Silva, R.; Martins, E.; Lobo, J. M. S.; Silva, A. C. Int. J. Pharm. 2017, 519, 373-380. doi:10.1016/j.ijpharm.2017.01.045

28. Castro, G. A.; Oréfice, R. L.; Vilela, J. M. C.; Andrade, M. S.; Ferreira, L. A. M. J. Microencapsulation 2007, 24, 395-407. doi:10.1080/02652040701288519

29. Kumar, S.; Randhawa, J. K. Mater. Sci. Eng., C 2013, 33, 1842-1852. doi:10.1016/j.msec.2013.01.037

30. Pardeike, J.; Hommoss, A.; Müller, R. H. Int. J. Pharm. 2009, 366, 170-184. doi:10.1016/j.jpharm.2008.10.003

31. Üner, M.; Yener, G. Int. J. Nanomed. 2007, 2, 289-300.

32. Jores, K.; Mehnert, W.; Drechsler, M.; Bunjes, H.; Johann, C.; Mäder, K. J. Controlled Release 2004, 95, 217-227. doi:10.1016/j.jconrel.2003.11.012

33. Müller, R. H.; Mäder, K.; Gohla, S. Eur. J. Pharm. Biopharm. 2000, 50, 161-177. doi:10.1016/s0939-6411(00)00087-4
34. Jenning, V.; Thünemann, A. F.; Gohla, S. H. Int. J. Pharm. 2000, 199, 167-177. doi:10.1016/s0378-5173(00)00378-1

35. Dingler, A.; Gohla, S. J. Microencapsulation 2002, 19, 11-16. doi:10.1080/02652040010018056

36. Paliwal, R.; Babu, R. J.; Palakurthi, S. AAPS PharmSciTech 2014, 15, 1527-1534. doi:10.1208/s12249-014-0177-9

37. Esposito, E.; Drechsler, M.; Mariani, P.; Carducci, F.; Servadio, M.; Melancia, F.; Ratano, P.; Campolongo, P.; Trezza, V.; Cortesi, R.; Nastruzzi, C. Biomed. Microdevices 2017, 19, 44-58. doi:10.1007/s10544-017-0188-x

38. Pecora, R. J. Nanopart. Res. 2000, 2, 123-131. doi:10.1023/a:1010067107182

39. Esposito, E.; Fantin, M.; Marti, M.; Drechsler, M.; Paccamiccio, L.; Mariani, P.; Sivieri, E.; Lain, F.; Menegatti, E.; Morari, M.; Cortesi, R. Pharm. Res. 2008, 25, 1521-1530. doi:10.1007/s11095-007-9514-y

40. Kulkarni, C. V. Nanoscale 2012, 4, 5779-5791. doi:10.1039/c2nr31465g

41. Puglia, C.; Bonina, F.; Rizza, L.; Cortesi, R.; Merlotti, E.; Drechsler, M.; Mariani, P.; Contado, C.; Ravani, L.; Esposito, E. J. Pharm. Sci. 2010, 99, 2819-2829. doi:10.1002/jps.22028

42. Pugh, W. J. In Aultons's Pharmaceutics-The design and manufacture of the medicines; Aulton, M. E., Ed.; Churchil Livingstone Elsevier: London, United Kingdom, 2007; pp 99-107.

43. Esposito, E.; Sticozzi, C.; Ravani, L.; Drechsler, M.; Muresan, X. M.; Cervellati, F.; Cortesi, R.; Valacchi, G. Exp. Dermatol. 2015, 24 , 449-454. doi:10.1111/exd.12696

44. Muresan, X. M.; Sticozzi, C.; Belmonte, G.; Savelli, V.; Evelson, P.; Valacchi, G. Mech. Ageing Dev. 2018, 172, 78-85. doi:10.1016/j.mad.2017.11.006

45. Muresan, X. M.; Sticozzi, C.; Belmonte, G.; Cervellati, F.; Ferrara, F.; Lila, M. A.; Valacchi, G. Arch. Biochem. Biophys. 2018, 658, 1-6. doi:10.1016/j.abb.2018.09.014

46. Valacchi, G.; Pecorelli, A.; Belmonte, G.; Pambianchi, E.; Cervellati, F.; Lynch, S.; Krol, Y.; Oresajo, C. J. Invest. Dermatol. 2017, 137, 1373-1375. doi:10.1016/j.jid.2017.01.034

47. Virtual Computational Chemistry Laboratory, ALOGPS 2.1; , http://www.vcclab.org/lab/alogps/.

48. NCBI homepage U.S.. National Library of Medicine National Center for Biotechnology Information: Bethesda, MA, U.S.A.https://pubchem.ncbi.nlm.nih.gov/compound/Retinoic-acid.

49. Abraham, N. G.; Kappas, A. Pharmacol. Rev. 2008, 60, 79-127. doi:10.1124/pr.107.07104

50. Atzori, L.; Caramori, G.; Lim, S.; Jazrawi, E.; Donnelly, L.; Adcock, I.; Barnes, P. J.; Chung, K. F. Respir. Med. 2004, 98, 530-535. doi:10.1016/j.rmed.2003.11.007 


\section{License and Terms}

This is an Open Access article under the terms of the Creative Commons Attribution License (http://creativecommons.org/licenses/by/4.0). Please note that the reuse, redistribution and reproduction in particular requires that the authors and source are credited.

The license is subject to the Beilstein Journal of Nanotechnology terms and conditions: (https://www.beilstein-journals.org/bjnano)

The definitive version of this article is the electronic one which can be found at:

doi:10.3762/bjnano.10.174 